\title{
Validity and Reliability Evidence of the Basic Psychological Needs in Exercise Scale (BPN-ES) in a European Sample of Individuals with Intellectual Disabilities
}

\author{
Skordilis Emmanouil, Greenlees Iain, Chrysagis Nikolaos, Grammatopoulou Eirini, \\ Papadopoulou Vasiliki, Pardo Gill Francisco Javier, Gaillard Joel, Skordilis Antonios, Dias Joao, \\ Serras Dionysios
}

\begin{abstract}
The study was based on the Self-Determination Theory (SDT) and examined the validity and reliability of the Basic Psychological Needs in Exercise Scale (BPN-ES)[27] of individuals with Intellectual Disabilities (ID) from Greece, Cyprus and Portugal. The participants were 152 adolescents and adults with ID, 72 females and 80 males, with high $(\mathrm{N}=92)$, moderate $(N=22)$ or low functionality $(N=38)$, aging 16 to 51 years old, attending daily centers in Greece $(\mathrm{N}=100)$, Portugal $(\mathrm{N}=20)$ and Cyprus $(\mathrm{N}=32)$. Data was collected from a psychologist and a coach, both employed at the respective daily centers. The statistical analyses revealed sufficient predictive validity evidence through the intercorrelations of the BPN-ES (autonomy, competence and relatedness) with the Pictorial Motivation Scale (intrinsic motivation - IM, self-determined extrinsic motivation - SDEM, non-self-determined extrinsic motivation - NSDEM, and amotivation - AM). Further, significant differences were found among individuals who differed according to functionality with respect to autonomy and competence (construct validity evidence). The BPN-ES Cronbach alpha indexes $(.703$ to .709$)$ and the Intraclass coefficients $\mathbf{( . 6 8 9}$ to .831$)$ provided sufficient internal consistency and test retest reliability evidence. The present findings supported the BPN-ES psychometrics for individuals with ID and are discussed accordingly. Certain limitations however, such as co morbidities, sampling methods, established golden standard, blind assessments and ethnic backgrounds do not allow generalization without caution. Future researchers may overcome these limitations and establish a solid motivation measure across Europe, based on the SDT, for individuals with ID.
\end{abstract}

Skordilis Emmanouil, National and Kapodistrian University of Athens, School of Physical Education and Sport Science, Greece

Greenlees Iain, Chichester Institute of Sport, University of Chichester, UK Chrysagis Nikolaos, National and Kapodistrian University of Athens, School of Physical Education and Sport Science, Greece Grammatopoulou Eirini, National and Kapodistrian University of Athens, School of Physical Education and Sport Science, Greece

Papadopoulou Vasiliki, Psychosocial Research Organization, Drapetsona, Piraeus, Greece

Pardo Gill Francisco Javier , Fundacion General De La Universidad Europea De Madrid, Spain

Gaillard Joel, Universite de Lorraine, France

Skordilis Antonios, Amfidromo Chorotheatro, Cyprus

Dias Joao, Associacao Para A Recuperacao De Cidadaos In Adaptados De Lousa, Portugal

Serras Dionysios, Psychosocial Research Organization, Drapetsona, Piraeus, Greece
Index Terms- autonomy-competence-relatedness, intellectual disabilities, physical activity, self-determination

Abbreviations: ID: Intellectual Disabilities, SDT: Self-Determination Theory, PA: Physical Activity, BPN-ES: Basic Psychological Needs in Exercise Scale, PMS: Pictorial Motivation Scale, IM: Intrinsic Motivation, SDEM: Self-Determined Extrinsic Motvation, NSDEM: Non-Self-Determined Extrinsic Motvation,, AM: Amotivation.

\section{INTRODUCTION}

Participation in regular exercise and physical activity (PA) among individuals with a disability is generally lower than that of their counterparts without a disability [1][2][3]. It is estimated that only $23 \%$ of individuals with disabilities in the USA engage in regular PA (20-30 minute daily) [4] and major reasons may be the exhibited barriers and their low motivation to participate [5].

With respect to individuals with intellectual disabilities (ID), researchers [6] claimed that their involvement in regular exercise and PA is less compared to their non disabled counterparts. Low engagement in PA is associated with obesity, cardiovascular and metabolic diseases [7], low muscle strength and endurance, and low exercise heart rate [8]. Further, females, at an older age, with more severe ID are at a higher risk for physical inactivity, obesity, cardiovascular and metabolic diseases [7]. Skowronski et al (2009) indicated that certain variables (e.g. strength, speed, flexibility, balance) may distinguish individuals with ID who differ according to gender, age and disability status (mild, moderate, severe) [9] while Phillips and Holland (2011) supported that the involvement in exercise and PA decreases in more severe levels of ID [10]. Individuals with ID and higher intellectual ability have, in general, fewer restrictions, less need for supervision, and more independence and motivation to exercise and be physically fit [10][11]. On the other hand, individuals with lower ability and more severe ID have the tendency to be more sedentary [12].

Exploring the barriers to involvement in recreation and PA may support the development of respective interventions designed to promote health and overall well-being in ID populations. Researchers claimed that fundamental reasons for the low involvement and physical activity levels of people with ID may be overprotection, inaccessible facilities and 
lack of opportunities [2][13], while others [6][14] argue that their well being is influenced from their personality, intelligence and general motivation to engage in daily activities. Edmond, Pelletier and Joussemet (2017) stated that motivation plays an important role for overcoming passivity and engagement in several activities [15] while Frielink et al. (2017) stated that ID populations are less motivated and more passive in several aspects of their lives than non-disabled counterparts [16]. Whilst it is important not to ignore the environmental barriers that may influence PA levels in ID populations, it is thus possible that research exploring motivational processes in ID populations will enhance understanding and lead to the development of interventions designed to enhance their PA levels.

The Self-Determination Theory (SDT) [17][18] is a universal motivation theory that offers a potential framework for understanding low levels of physical activity in ID populations. There are four key SDT propositions guiding human action and functioning, all of which have been supported by research evidence across a variety of life domains (e.g. sport, education, employment) and populations (e.g. cultures, disabilities). The first proposition suggests that individuals differ in the reasons why they invest effort, persist and/or withdraw from several activities. Individuals who are more self-determined (or autonomous) engage in an activity because of the enjoyment and pleasure they derive from the activity (intrinsic motivation), perceive the activity to be part of their identity (integrated regulation), or value the activity for the benefits it brings (identified regulation). On the other hand, individuals who are non-self-determined participate in activities because of external rewards, inducements or coercion (extrinsic motivation), to avoid negative emotions (e.g. shame) associated with not doing the activity (introjected regulation). The second proposition suggests that individuals lie on a continuum from self-determined (autonomous) to non-self-determined (controlled) motivation and finally to amotivation [19]. More self-determined forms of motivation are associated with increased effort, persistence, skill development, lower anxiety, better performance [20] and enhanced psychological health and well-being [21]. The third proposition introduces the three major determinants of the self-determined form of motivation. According to researchers [19][22], self-determined motivation for a certain activity is determined by the satisfaction of three basic human needs: a) autonomy (need to feel in control of our choices), b) competence (need to feel we are good at an activity and accomplish challenges) and c) relatedness (need to feel connected and experience a sense of sharing to others. The fourth and final proposition claims that social environments, and the individuals managing these environments, exert an important influence on whether needs are thwarted or met [19]. In exercise and sports, for example, the coaches may involve their athletes in the decision making roles, offer strategies for self-improvement (autonomy), provide performance and task-related feedback (competence) and develop social, task-related relationships in a positive team-climate environment (relatedness) [22].

With respect to individuals with ID, previous researchers have successfully applied SDT across several domains, including recreation, leisure, sport and physical activity (PA). Edmond Pelletier and Joussemet (2017) provided the first evidence that autonomy supported leisure interventions could be effective in enhancing self-determined motivation in individuals diagnosed with ID [15]. The researchers concluded that these interventions may exert positive and meaningful changes even after a single session [15]. Farrell, Crocker, McDonough and Sedgwick (2004) examined Special Olympians' perceptions of motivation and found support for the role of autonomy, competence and relatedness in determining motivation [6]. Participants claimed that relatedness was the central determinant of motivation and reported that they felt especially motivated when receiving social support and encouragement from coaches, teammates and parents. The researchers concluded that relatedness may be particularly important for individuals with ID due to impoverished friendship networks [6]. Goodwin et al. (2006)[23] examined the decision making experiences of the parents of athletes with ID in the Special Olympics [23]. The researchers claimed that long-term involvement in sports is associated with intrinsic motivation, independence, perceived competence, goal-setting and self-awareness. Goodwin et al claimed that the parental role is essential in the development of self-determined behaviors of their children with developmental disabilities in sports [23]. The researchers found that parents were eager for their children to participate in teams with coaches building bonds and relationships with their team members. Further, the anxiety-free environment was important and the parents gave credit to instructors and environments that provide peer interactions, increase independence and clear expectations for meaningful motor skill development [23]. Rossow-Kimball and Goodwin (2009) examined the leisure experiences of women with ID living in residential group homes [24]. The researchers claimed that choice making (such as in leisure activities) is essential to reach self-determination and found that the experience of leisure was different in each home setting, mainly due to the staff involved and the choices they provided to the women with ID. In one of the group homes for example, the leisure time was directional and supervised, while independent leisure was encouraged only in the second home where staff members encouraged personal choice, autonomy, decision making and spontaneity [24]. Finally, Hutzler, et al. [8] examined the sport motivation and goal perspectives of Special Olympic athletes and athletes without disabilities. The researchers found that the athletes with ID scored higher in the majority of the motivational scales compared to their non ID counterparts. Further, the external motivation of the ID group increased across time while the opposite was observed in the non ID group. Hutzler et al. [8] concluded that these differences may be considered by practitioners in the field who are planning training and competitions for individuals with ID.

Whilst research does seem to testify to the relevance of SDT principles in ID populations, a fuller examination of the theory's relevance to explaining physical activity levels is dependent on the development of validated measures of its central constructs (i.e., forms of motivation and basic need 
satisfaction). A variety of scales have been used to assess basic SDT elements in general populations. The validity and reliability of these scales have been examined in several countries (e.g. Canada, USA, UK, Italy, Greece, Spain, France, Netherlands, New Zealand) and samples (e.g. athletes, elderly, university students, elementary and high school students, exercisers) so far and appeared in the literature as Interpersonal Support in Physical Activity Consultations Observational Tool - ISPACOT [25], Perceived Locus of Causality Scale - PLOC [26], Basic Psychological Needs in Exercise Scale - BPN - ES and Basic Psychological Needs in Physical Education - BPN - PE [27][28] Psychological Need Satisfaction in Exercise Scale PNSE [29] Perceived Autonomy Support Scale for Exercise Settings - PASSES [30], Sport Motivation Scale - SMS [31], Behavioral Regulation in Exercise Questionnaire - BREQ [32], Fitness Actions and Dispositions Questionnaire - FADQ [33], and Self Regulation Questionnaire - SRQ [34]. Further, they have been used to assess individuals with a variety of disabilities, such as physical disabilities [35][36][37][38][39] learning disabilities [40], autism spectrum disorder - ASD [41][42] and intellectual disabilities - ID [16][43][44][45].

Our literature review suggested that the validity and reliability of the scales used to examine the SDT upon individuals with ID has received limited research attention so far [14][16][44][46][47][48]. Reid et al [47] developed and validated the Pictorial Motivation Scale (PMS) to evaluate motivation toward sport and physical activity of individuals with ID in Canada. The PMS Scale assesses contextual motivation toward sport and physical activity in 16 items, presented in a 3 - point pictorial form ('like me', somewhat like me', not like me'), under four factors named intrinsic motivation, self-determined extrinsic motivation, non-self-determined extrinsic motivation and amotivation. The researchers claimed that the PMS was the only multidimensional pictorial type scale so far to record the intrinsic and extrinsic motivation of individuals with ID in the sport and physical activity contexts [47]. Sajute [48] developed the Lithuanian version of the Sport Motivation Scale - SMS for individuals with ID. The Lithuanian SMS consisted from 16 items, rated on a 3 - point pictorial scale, under the subscales of intrinsic motivation, extrinsic motivation and amotivation. Sajute [48] claimed that the 3 -point pictorial form, ranging from not true (1) to true (3), was adopted to assist the participants to understand the statements and the respective responses. Sexton et al [44] examined the concurrent validity of emotional well-being and self-determination scales for adults with and without ID in Ireland. The researchers found weak concurrent validity evidence between self-determination and well-being measures and concluded that specific instruments, valid and feasible to use, are required to examine and compare individuals with and without ID [44]. Frielink et al [16] examined the universality of the extrinsic motivation subtypes, in adults with mild and borderline ID in the Netherlands. Specifically, they examined the extent to which Deci and Ryan's [49] extrinsic motivation type continuum are experienced from individuals with ID. The researchers stated that the analyses supported their hypothesis, provided the basis for using the self-determination principles in samples of individuals with ID, but claimed that more research is required to present construct validity evidence and improve the reliability of the measures employed [16]. Frielink et al. [46] examined the satisfaction of basic needs among individuals with ID living with residential daily support. The researchers found an adequate fit for the tested model and stated that the psychological needs for autonomy, relatedness, and competence are important, interrelated concepts for people with ID with borderline and mild ID [46]. Finally, Katz and Cohen [14] examined the validity evidence of the relative autonomous motivation instrument, in Israeli students with impaired cognitive abilities [14]. The researchers stated that the intercorrelations among the respective variables (relative, autonomous and controlled motivation) supported the predictive validity but claimed that the validity of the instrument should be assessed against other instruments to avoid the questionable validity problems of self-report questionnaires [14].

Summarizing our literature review, it appears that SDT and the scales used to examine it's different constructs (motivation levels and basic needs) have been examined from several researchers, across a variety of domains and populations in the past. The research evidence however is limited with regards to measures of basic needs satisfaction for use with individuals with ID. The present study, therefore, was designed to provide validity and reliability evidence for the Basic Psychological Needs in Exercise Scale (BPN - ES) [27][28] in a sample of individuals with ID, from three separate European countries (Greece, Cyprus and Portugal). Based on our literature review and published guidelines [50][51][52] we anticipated sufficient predictive validity, construct validity and reliability evidence (test retest and internal consistency). For the purposes of the present study, predictive validity referred to the association between test scores (BPN - ES) and the established criterion (PMS) and construct validity referred to group differences [50]. Test retest referred to the consistency of a measurement across time [53] and internal consistency to the reliability of a scale according to the degree of associations within the respective items [54]. Specifically, we anticipated that the BSN - ES factors would: a) correlate with the PMS factors of Reid et al [55] (predictive validity) such that positive associations should emerge between the three basic needs and self-determined motivation and negative associations should emerge between the basic needs and non-self-determined forms of motivation and with amotivation, b) distinguish ID athletes with high, moderate and low functionality (construct validity) and c) exhibit appropriate Cronbach alpha and Intraclass reliability coefficients.

\section{METHOD}

\section{Participants}

A purposive sampling design was introduced in the present study. The participants were adolescents and adults diagnosed with intellectual disability (ID) in their respective countries and were receiving residential day support. All participants had been living with their families during the six months prior to data collection. The definition of the 
American Psychiatric Association [56] describes ID as "intellectual and adaptive functioning deficits in conceptual, social, and practical domains" (p. 37). Support was defined in the present study as the strategies involved and the resources used to enhance human functioning [57]. The day support was provided by certain daily centers engaging and specializing in the treatment and rehabilitation of individuals with ID. These centers offer daily activities such as physical and occupational therapy, exercise and physical activities, and teach occupational, social and adaptive skills. Their main goal was to maximize the functionality of the individuals receiving their services. The information with respect to functionality (high, moderate, low), was provided by two staff members employed in the daily centers, according to pre determined criteria [58] and followed criteria adopted by previous researchers [46].

The total sample constituted from 152 participants, 72 females and 80 males, ranging in age from 16 to 51 years (M $=26.87, \mathrm{SD}=7.06)$. The participants were attending centers with daily activities in Greece $(\mathrm{N}=100,65.79 \%)$, Portugal $(\mathrm{N}=20,13.16 \%)$ and Cyprus $(\mathrm{N}=32,21.05 \%)$, and exhibited, according to the responsible staff members, either high $(\mathrm{N}=92,60.53 \%)$, moderate $(\mathrm{N}=22,14.47 \%)$ or low functionality $(\mathrm{N}=38,25 \%)$. Seventy two declared they had practiced exercise and physical activity in the past and the vast majority reported they had lived in their family home for the whole duration of their lives (only 3 participants declared they were living alone at sometime in the past).

\section{MEASURING InSTRUMENTS}

The Basic Psychological Needs in Exercise Scale (BPN ES)[27] was used as the primary variable for the purposes of the study. The scale was selected as it has previously been validated in a wide sample of Greek adults, aging 18 to 64 years old. Vlachopoulos and Michailidou [27] found that the scale was invariant across separate samples which differed according to gender and participation in organized physical activity, and exhibited acceptable reliability indexes. The BPN - ES incorporates 12 items, classified under the three factors of Autonomy, Competence and Relatedness, and the responses are provided in a 7 Likert type scale, from strongly disagree (1) to strongly agree (5). In general, higher scores (close to 5) indicate higher levels of autonomy, competence and relatedness. Item examples are: a) 'I feel that I have the opportunity to make choices with respect to the way I exercise' (autonomy), b) 'I feel that exercise is an activity in which I do very well' (competence) and c) 'I feel comfortable with the other exercise participants' (relatedness). For the purposes of the present study and following the guidelines of previous researchers [47, 59] the following steps were undertaken in each country: 1) The items were rewritten in an interrogative and exemplified form and the original 7-point Likert-type response options were simplified in a 3-point pictorial format. Lower scores were indicative of wider satisfaction of the three basic needs for autonomy, competence and relatedness. 2) The researchers showed pictures with variations of the 'happy face' to a pilot sample of five individuals with mild ID who, in turn, responded to the research team and indicated whether the situations depicted in the pictures were 'not like me' (recorded as 3), 'a little bit like me' (recorded as 2), and 'like me' (recorded as 1). The administration required approximately 10 minutes to finish and no difficulties were recorded from the researchers. Accordingly, 3) our research team adopted the pictorial format and introduced pictures and phrases in each factor to assess our main sample of individuals with ID.

The Pictorial Motivation Scale (PMS) [47] was used as the criterion (golden standard) to establish predictive validity. The PMS assesses four types (dimensions) of contextual motivation for sports and physical activity of individuals with ID (intrinsic motivation - IM, self-determined extrinsic motivation - SDEM, non self-determined extrinsic motivation - NSDEM, and amotivation - AM). The PMS was established according to the hierarchical motivation model (intrinsic and extrinsic) of Vallerand and associates [60- 62], and is congruent with the viewpoints of self-determination by Deci and Ryan [17] and Wehmeyer [63]. Each dimension of the scale incorporates four pictures (items) and phrases. During data collection, the administrators present the pictures to the participant and read the phrase associated with them. The respondents, in turn, indicate if the situation depicted is 'like me' (recorded as 3), 'a little bit like me' (recorded as 2), and 'not like me' (recorded as 1). In general, higher scores (close to 3) indicate higher levels of IM, SDEM, NSDEM and AM respectively. Examples of items are: I participate in sports a) 'because it is fun' (IM), b) 'because it is a good way to learn things which can be useful in life' (SDEM), c) 'to please my parents or my coach' (NSDEM), and d) 'but I feel that I am wasting my time' (AM).

A demographic questionnaire was used to gather general information with respect to age, gender, residence, functionality, employment, family income, athletic experience, etc. In certain questions, if the participants had difficulty to provide the respective information, the research team asked for their permission to address the staff member who was usually present during the data collection.

\section{PROCEDURE}

The data was collected as a part of a wider study funded by the European Union. The aim of the present study was to provide validity and reliability evidence of the BPN - ES in three separate samples of individuals with ID across Greece, Cyprus and Portugal. The wider study was a randomized controlled trial comparing the effect of a 9 month organized exercise program, based on the SDT principles, upon the physical conditioning and motivation of individuals with ID.

Prior to data collection, a research team consisting of a psychologist and a coach, both experienced with ID populations and employed at the respective daily centers, met with their fellow administrators, staff and the individuals with ID receiving their services. The research team: a) explained the purpose of the study and b) asked for permission to administer the BPN - ES, PMS and the demographic questionnaires. The participants who declared their willingness to participate, whose functionality was recorded by the staff members and were able to understand and follow simple instructions constituted the total sample. 


\section{ISSN:2455-3956, Volume-8, Issue-6, June 2019 Pages 06-15}

The data collection was held at the respective daily centers, at noon, in a well known environment (e.g. room for adaptive games and group activities). The psychologist and the coach prepared the instruments with all the necessary material. The participants entered the data collection area along with a responsible staff member and signed the informed consent. The staff member was allowed to stay and facilitate the data collection process. Finally, a limited number of participants from Greece $(\mathrm{N}=30)$, were re assessed $10-15$ days later, to confirm the reliability of the responses.

With respect to the adopted translation validity method by the three European countries involved (Greece, Cyprus and Portugal), the US Census Bureau [64] recommended procedures for translating questionnaires was used for all measures. An overview of the method and criteria for proper translations is presented in the Census Bureau website and is summarized in a 5 step protocol: Prepare, Translate, Pretest, Revise and Document. The Census Bureau does not recommend solo or direct translation with back translation, but promotes a translation process and review by a team of translators, reviewers and adjudicators. As preparation, translators were supplied with a summary of the scope of the whole project, information of the target population, survey documentation with definitions of terms or concepts and access to experts who may assist them. Pretesting is a necessary step that identifies problems in the translated text or helps identify other concepts that may be relevant within the target population. Documentation of the translation process at each step makes it possible to track the different survey versions or demonstrate that the survey functions well in the pretests. The guidelines of the Census Bureau stem from a meeting of experts, which was designed, sponsored, and hosted by the Census Bureau in November 2001. Further, the documents on the website provide practical guidance and considerations (geographic location, social and cultural factors) that will impact the translation process.

The psychologist and the coach employed at the daily centers were responsible for data collection. They all had a minimum experience of five years of working, training and coaching individuals with ID. The aim was to administer the questionnaires individually, following the guidelines of Reid and associates [47][55]. The participants initially spent a few minutes with the assessors in order to relax and familiarize themselves with the procedure, the pictorial scales, and the respective responding options ('like me', 'a little bit like me', and 'not like me'). Accordingly, the assessors were instructed to ensure, as much as they could, that the participants were comfortable, understood the questions being asked and enjoyed the whole process. Only the data from participants who demonstrated sufficient comprehension of the questions were incorporated in the present study. Participants were offered the opportunity to attend the testing session with a relative, friend or support worker.

\section{Statistical ANALYSES}

The SPSS for Windows (version 18.0) was used for statistical analyses. Initial screening of the data was performed with pre determined criteria (skeweness $>2.0$, kyrtosis > 3.2, Kolmogorov Smirnov with p > .05) [29]. Intra rater reliability was examined with the Intraclass coefficients (ICC) and internal consistency with the Cronbach alpha coefficients [53][ 65]. The recommendations of Shrout [66] were followed, who claimed that reliability indexes $<.10$ is virtually none, .11 to .40 is slight, .41 to .60 is fair, .61 to .80 is moderate and finally .81 to 1.00 is substantial. The Spearman intercorrelation coefficients were used to provide predictive validity evidence for the BPN - ES. The PMS was used as the criterion variable and intercorrelation coefficients above .50 were moderate - high, .30 - .50 were moderate, .20 - . 30 were low - moderate and below .20 were low [67]. Finally, three ANOVAs were employed to provide construct validity evidence. The ANOVAs examined the differences of individuals with ID who differed according to functionality (high, moderate, low) in the three BPN - ES factors (Autonomy, Competence, Relatedness). The Least Significance Difference (LSD) method was used for post hoc comparisons [68].

\section{RESULTS}

The responses of the participants in the three BPN - ES and the four PMS factors are presented in table 1. The Intraclass (ICC) and Cronbach alpha coefficients examining the intra rater reliability and internal consistency are presented in table 2. The Spearman intercorrelations between the BPN - ES and the PMS factors were used to provide predictive validity evidence and are presented in table 3 . Finally, three separate ANOVAs examined the differences of individuals with ID who differed according to functionality (high, moderate, low) in the three BPN - ES factors (Autonomy, Competence, Relatedness). The results were significant for Autonomy $(\mathrm{F}=2.997, \mathrm{p}=.039, \eta 2=.058)$ and Competence $(\mathrm{F}=4.461, \mathrm{p}=.014,=\eta 2=.078)$ but not for Relatedness $(\mathrm{F}=.599, \mathrm{p}=.551, \eta 2=.011)$. With respect to Autonomy, the LSD post hoc comparisons revealed that the high functionality group scored lower compared to the low $(\mathrm{MD}=-.28, \mathrm{p}=.020)$ group. With respect to Competence, the LSD revealed that the high functionality group scored lower compared to the low (MD $=-.39, \mathrm{p}=.007)$ group. The findings are presented in figures 1 and 2 .

Table 1 The responses of individuals with ID (high, moderate and low functionality) in the three BPN - ES and the four PMS factors

\begin{tabular}{|c|c|c|c|c|}
\hline Variable & $\begin{array}{l}\text { Total } \\
\text { Sample }\end{array}$ & High & Moderate & \\
\hline $\begin{array}{l}\text { BPN - ES } \\
\text { Autonomy }\end{array}$ & & & & \\
\hline Competence & $2.56 \pm .50$ & $2.48 \pm .48$ & $2.61 \pm .50$ & $2.76 \pm .51$ \\
\hline & $2.44 \pm .60$ & $2.32 \pm .61$ & $2.59 \pm .62$ & $2.71 \pm .43$ \\
\hline Relatedness & $2.76 \pm .43$ & $2.73 \pm .44$ & $2.80 \pm .41$ & $2.84 \underline{+} .42$ \\
\hline $\begin{array}{l}\mathrm{IM}^{1} \\
\mathrm{SDEM}^{2} \\
\mathrm{NSDEN} \\
\mathrm{AM}^{4}\end{array}$ & $\begin{array}{c}1.37 \pm .42 \\
1.30 \pm .43 \\
\mathrm{M}^{3} 1.71 \pm .68 \\
2.41 \pm .50\end{array}$ & $\begin{array}{c}1.42 \pm .44 \\
1.33 \pm .44 \\
1.86 \pm .72 \\
2.43 \pm .50\end{array}$ & $\begin{array}{c}1.16 \pm .29 \\
1.21 \pm .44 \\
1.37 \pm .58 \\
2.44 \pm .34\end{array}$ & $\begin{array}{c}1.30 \pm .40 \\
1.27 \pm .38 \\
1.50 \pm .49 \\
2.35 \pm .58\end{array}$ \\
\hline
\end{tabular}

1: Internal motivation, 2: Self-determined external motivation, 3: Non-self-determined external motivation, 4: Amotivation 
Table 2 Intraclass (ICC) and Cronbach alpha reliability coefficients, in the three BPN - ES and the four PMS factors

\begin{tabular}{|c|c|c|}
\hline Variable & ICC & Cronbach \\
\hline \multicolumn{3}{|l|}{$\overline{\text { BPN - ES }}$} \\
\hline Autonomy & .742 & .703 \\
\hline Competence & .831 & .791 \\
\hline Relatedness & .689 & .779 \\
\hline \multicolumn{3}{|l|}{ PMS } \\
\hline $\mathrm{IM}^{1}$ & .741 & .489 \\
\hline SDEM $^{2}$ & .742 & .587 \\
\hline NSDEM $^{3}$ & .893 & .775 \\
\hline $\mathrm{AM}^{4}$ & .102 & .506 \\
\hline
\end{tabular}

1: Internal motivation, 2: Self-determined external motivation, 3: Non-self-determined external motivation, 4: Amotivation

Table 3 Intercorrelation matrix between the BPN - ES and the PMS factors

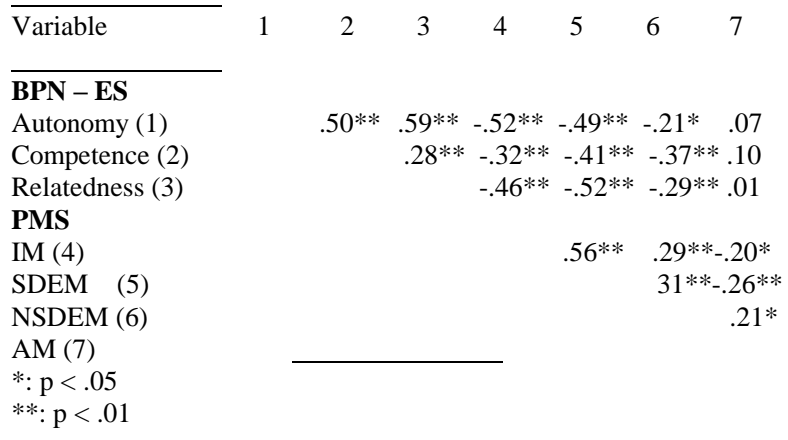

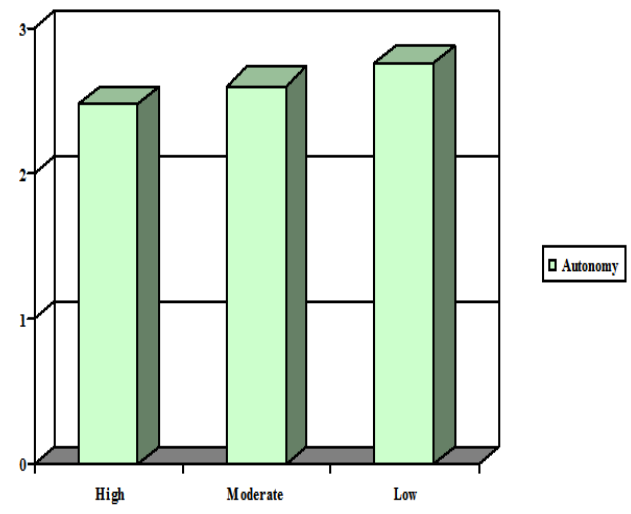

Fig. 1 Differences of individuals with ID who differed according to functionality (high, moderate, low) in Autonomy $\left(\mathrm{F}=2.997, \mathrm{p}=.039, \eta^{2}=\right.$ .058) (LSD post hoc: high < low)

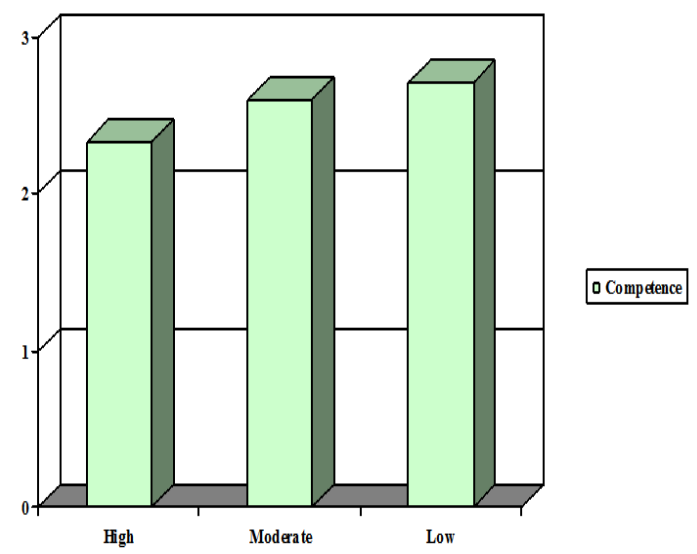

Fig. 2 Differences of individuals with ID who differed according to functionality (high, moderate, low) in Competence $\left(\mathrm{F}=4.461, \mathrm{p}=.014, \eta^{2}=\right.$ .078) (LSD post hoc: high < low)

\section{DISCUSSION}

The present study attempted to provide validity (predictive and construct) and reliability (test retest and internal consistency) evidence for the Basic Psychological Needs in Exercise Scale (BPN-ES) in a European Sample of individuals with Intellectual Disabilities (ID). We followed the guidelines provided by researchers [50][69] who disputed the traditional views and recommended that validity evidence must be reported constantly. The predictive validity evidence was provided through the intercorrelations of the BPN - ES with the PMS criterion. The construct validity evidence was provided through the differences examined among ID individuals who differed according to functionality.

Finally, the test - retest and internal consistency analyses were used to provide reliability evidence [51].

The present findings provided sufficient predictive validity evidence, are in agreement with Reid et al [47], Frielink et al [16][46] and Katz and Cohen [14] and in conflict with Sexton et al [44]. Sexton et al [44] stated that several measures of self determination exhibited limited evidence of construct and predictive validity and concluded that valid and appropriate self-determination measures in the ID population are crucial to design and evaluate policies related to health. On the other hand, Reid et al [47] provided concurrent validity evidence through support for the theoretically hypothesized correlation pattern among the four subscales (IM, SDEM, NSDEM and AM). Reid et al [47] stated that the need for competence correlated positively with IM and negative with AM, and concluded that future validation research may examine the association of the PMS to autonomy, relatedness, and outcomes such as persistence and performance. Similarly, Frielink et al [16] found a pattern of intercorrelations, with adjacent types of extrinsic motivation related more highly than non adjacent types. The researchers stated that the intercorrelation indexes recorded among the adjacent factors (external, introjected, identified and integrated motivation) were sufficient for early stage research (.50 to .60) [16]. Frielink et al [46] hypothesized that satisfaction of autonomy, competence and relatedness would relate positively to autonomous motivation and negative to controlled motivation. The researchers found a significant inter-correlation only between relatedness and autonomous motivation and claimed that individuals with ID may perceive autonomy as independence. In that case, they do not experience autonomous motivation, even when their psychological needs are met [46]. Katz and Cohen [14] examined initially the intercorrelations among the elements of the relative autonomous motivation scale used and secondly, their respective associations to other constructs. The researchers found a positive intercorrelation of autonomous motivation to positive affect and task value, and a negative intercorrelation between the autonomous and the controlled elements of the scale used. Katz and Cohen [14] concluded that their research evidence supported the validity of the autonomous motivation scale for individuals with cognitive impairments [14].

The present findings suggested that the BPN - ES subscales: a) associated significantly with each other and, in accord with theoretical predictions, b) exhibited a descending association 


\section{ISSN:2455-3956, Volume-8, Issue-6, June 2019 Pages 06-15}

pattern with the PMS subscales. In addition, the results indicated that there were stronger associations between the extent to which basic needs were satisfied and self-determined forms of motivation (IM and SDEM) than between AM and NSDEM. Whilst these findings indicate general support for the BPN-ES, there were some unexpected results. Specifically, we did not expect to see positive associations between the satisfaction of needs and non-self-determined forms of motivation as, theoretically, the NSDEM form should be associated with the non-satisfaction of basic needs. Whilst this was surprising, it may reflect the nature of the questions used in the PMS to assess NSDEM. The PMS NSDEM questions are concerned with doing an activity to gain attention/please others (parents, teachers and friends) and to demonstrate competence in a task to others. Whilst this does reflect some non-self-determined forms of motivation, it does not fully capture external regulation (doing an activity solely for tangible rewards and payment) or introjected regulation (doing an activity out of a sense of obligation to others or to self). To this end, the PMS may not fully tap into the range of motivation types that constitute NSDEM. However, whilst some degree of caution should be exercised when using the PMS, it does still appear to distinguish properly between states of amotivation and self-determined forms of motivation.

The mean competence scores, lower than autonomy and relatedness, revealed a consistent pattern across individuals with ID with either high, moderate or low functionality. This finding is consistent with previous reports of individuals with intellectual disabilities. Specifically, Farrell et al. [6] found evidence, in their qualitative exploration of determinants of self-determined motivation in Special Olympians that relatedness and social support (from peers, teachers, coaches, parents and team-mates) were especially important sources of motivation. In addition, Požérienè et al. [45] also testified to the importance of perceptions of relatedness in populations with intellectual disabilities. Thus, it may be that relatedness and the need to feel connection with others is a particularly powerful source by which activities come to be enjoyed and valued within this population. This information may be especially relevant to practitioners working with people with ID and seeking to enhance motivation to engage in physical activity that may have psychological and physiological benefits. Whilst many interventions designed to enhance self-determined forms of motivation in populations without intellectual disabilities stress the role of autonomy and competence [70] interventions that seek to also harness relatedness, social support and group connection/cohesion may be much more effective.

With respect to the construct validity hypothesis, the present findings provided evidence for the Autonomy and Competence subscales of the BPN - ES. Specifically, a) the mean scores of the autonomy and competence needs exhibited an increment across the three functional groups and b) this increment differentiated significantly the participants with high and low functionality. This finding is in agreement with Phillips and Holland [10] who stated that individuals with higher functionality experience less restrictions and supervision, and higher independence and motivation to address PA programs compared to their lower functioning counterparts. Hutzler and Korsensky [71] stated that one major barrier for the engagement in PA programs across time is the lack of motivation, while Burton-Smith [11] claimed that limited opportunities for self-determination throughout their lives are experienced from individuals with ID and more severe levels of disability. Chatzisarantis and Hagger [72] suggested that the extent of cognitive deficits upon individuals with ID is associated to their respective self-determination while Trost et al [73] stated that the engagement in PA of individuals with ID is related upon the severity and respective diagnosis. Rossow-Kimball and Goodwin [24] stated that even activities in leisure context (i.e. PA) may become a 'distancer', with a feeling of isolated 'disability hierarchy', as the level of impairment increases. Finally, several researchers claimed that the eagerness of individuals with ID to exercise self-determination as productive and integrated members of society is influenced from their health, which is related to their involvement in PA which, in turn, is related to their respective functional severity [74][75][76].

The Relatedness subscale did not differentiate the participants who differed according to their respective functionality (high, moderate, low). There was an increment however of the respective mean scores across the three groups, suggesting that individuals with ID and higher functionality experienced wider satisfaction through relatedness when involved in PA, compared to their counterparts with lower functionality. This increment though was not enough to substantiate significant differences and support our research hypothesis. One reason may be the absence of power analysis which, in accordance to the limited sample size of the participants in the moderate group $(\mathrm{N}=$ 22), did not guide the respective statistical analysis. Another explanation may be supported from the variability of the examined sample. According to O'Brien [77], most studies usually incorporate ID volunteers with mild to moderate cognitive delays. Individuals with severe ID (low functionality) may experience co-occurring medical and mobility restrictions, which make participation in PA and establishment of respective social bonds even more difficult [78]. Our data suggests that very few participants in the low functionality group had access to organized sports and PA programs, such as the MATP of the Special Olympics, giving more ground therefore to the above explanation.

The reliability coefficients of the BPN - ES in the present study (Intraclass and Cronbach alpha) are comparable to those reported in the ID literature [8][16][46] and within the moderate level of .61 to .80 , according to the criteria presented by Shrout [66]. The Cronbach alpha (internal consistency) coefficients ranged from .703 (Autonomy) to .791 (Competence), and the Intraclass (test retest) from .689 (Relatedness) to .742

(Autonomy). Only the Intraclass coefficient for Competence (.831) was perceived as substantial according to Shrout [66]. Similarly, Nunually et al [79] reported that reliability values between .50 and .60 are sufficient for early stages research, but values above .80 should be pursued at a later stage. Frielink et al [16] reported Cronbach alpha raging 
from .56 to .91 and Intraclass coefficients from .54 to .78 , Frielink et al [46] reported Cronbach alpha from .56 to .75 and Intraclass from .62 to .77 while Hutzler et al [8] reported Cronbach alpha from .81 (Internal motivation) to .31 (Amotivation). In the present study, low test retest reliability was reported only for Amotivation, a subscale of the criterion PMS, similarly to Poulin ([80] Hutzler et al [8] and Reid et al [47]). Poulin ([80] reported that only the amotivational subscale had marginal reliability, while Cronbach alpha coefficients ranged from .53 to .85 , and test retest from .61 to 94. Reid et al [47] stated that amotivation had the lowest test retest reliability $(.57)$ and the researchers must be careful when they interpret the respective findings. Reid et al [47] added that due to the moderate reliability, individuals with ID may receive some preparation training and practice before completing the PMS to ensure that they understand the pictorial response format ('like me', 'a little bit like me', 'not like me').

\section{CONCLUSION}

In general, the present findings supported our research hypotheses. The BPN - ES may be used with more confidence in the future to assess individuals with ID. Certain limitations, however, do not allow for generalization without caution. First, no co morbid conditions were reported, especially for the low functionality group of individuals with ID. Second, the sample was not stratified according to gender and severity. An invariance testing in the future would be useful to confirm the applicability of the BPN - ES across males and females, with high, moderate or low functionality. Third, the predictive validity was established through the intercorrelation with the PMS [47]. The PMS was based on the hierarchical model of intrinsic and extrinsic motivation developed by Vallerand and associates [60][61][62]. The $\mathrm{BPN}-\mathrm{ES}$ on the other hand was established from the basic psychological needs for autonomy, competence, and relatedness, which are perceived as essential elements to enhance motivation and psychological growth in every domain, including PA and sports [17][49]. This 'slight theoretical deviation' was inevitable, and the PMS was perceived as a solid measure to fit the demands of the 'golden standard' and support our effort to provide predictive validity evidence. This 'slight deviation' for instance may have contributed to the reported wider association of competence and relatedness to SDEM compared to the IM. Fourth, several aspects of behaviour, such as empowerment, quality of life, depression and anxiety were not examined in accordance to the BPN - ES and in an attempt to provide further validity evidence [71]. Fifth, the researchers responsible for data collection were not blind to the purposes of the study. Their differences therefore, with respect to background and training, may have unintentionally affected the responses of the participants with ID. Finally, we are aware that self determination may be perceived distinctively across different cultures [1]. The present findings therefore may not generalize to other countries, besides Greece, Cyprus and Portugal. Future researchers may attempt to overcome this limitation, by providing solid validity and reliability evidence of a single measure assessing the SDT principles of individuals with ID throughout Europe.

\section{ACKNOWLEDGEMENTS}

The study was co-funded by the Erasmus+ Programme of the European Union.

\section{CONFlict OF INTEREST}

The authors declare no conflict of interest

\section{REFERENCES}

[1] MacDonald C, Reid G. Instructional strategies of inclusive physical education teachers: Development and validation of a self-determination scale. European Journal of Adapted Physical Activity. 2013; 6: 43-56.

[2] Frey G, Stanish H, Temple V. Physical activity of youth with intellectual disability. Review and research agenda. Adapted Physical Activity Quarterly. 2008; 25: 95-117.

[3] Rimmer J, Rowland J. Health promotion for people with disabilities: Implications for empowering the person and promoting disability-friendly environments. American Journal of Lifestyle Medicine. 2008; 2: 409-420.

[4] U.S. Department of Health and Human Services. Physical activity and health: A report of the Surgeon General. Atlanta, Georgia: US Department of Health and Human Services, Public Health Service, CDC, National Centre for Chronic Disease Prevention and Health Promotion. 2000.

[5] Kosma M, Cardinal B, Rintala P. Motivating individuals with disabilities to be physically active. Quest. 2002; 54: 116-132.

[6] Farrell RJ, Crocker PJ, McDonough MH, Sedgwick WA. The driving force: Motivation in Special Olympians. Adapted Physical Activity Quarterly. 2004; 21: 153-166.

[7] Emerson E. Underweight, obesity and exercise among adults with intellectual disabilities in supported accommodation in Northern England. Journal of Intellectual Disability Research. 2005; 49 : 134-143.

[8] Hutzler Y, Oz M, Barak S. Goal perspectives and sport participation motivation of Special Olympians and typically developing athletes. Research in Developmental Disabilities. 2013; 34: 2149-2160.

[9] Skowronski W, Horvat M, Nocera J, Roswal G, Croce R. Eurofit special: European fitness battery score variation among individuals with intellectual disability. Adapted Physical Activity Quarterly. 2009; 26: 54-67.

[10] Phillips A, Holland A. Assessment of objectively measured physical activity levels in individuals with intellectual disability with and without Down's syndrome. PLOS ONE. 2011; 6(12): e28618.

[11] Burton Smith R, Morgan M, Davidson J. Does the daily choice making of adults with intellectual disability meet the normalisation principle? Journal of Intellectual \& Developmental Disability. 2005; 30: 226-235.

[12] Sherrill C. Adapted physical activity: Crossdisciplinary and lifespan 6th ed. Boston, MA: WCB/ McGraw-Hill. 2004.

[13] Sharav T, Bowman T. Dietary practices, physical activity, and body mass index in a selected population of Down syndrome children and their siblings. Clinical Pediatrics. 1992; 3: 341-344.

[14] Katz I, Cohen R. Assessing autonomous motivation in students with cognitive impairment. Journal of Intellectual \& Developmental Disability. 2014; 39: 323-332.

[15] Edmond Pelletier J, Joussemet M. The benefits of supporting the autonomy of individuals with mild intellectual disabilities: an experimental study. Journal of Applied Research in Intellectual Disabilities. 2017; 30: 830-846.

[16] Frielink N, Schuengel C, Embregts P. Distinguishing subtypes of extrinsic motivation among people with mild to borderline intellectual disability. Journal of Intellectual Disability Research. 2017; 61: 625-636.

[17] Deci EL, Ryan RM. Intrinsic motivation and self-determination in human behavior. New York: Freeman. 1985.

[18] Ryan RM, Deci EL. Self-determination theory and the facilitation of intrinsic motivation, social development and well-being. American Psychologist. 2000; 55: 68-78.

[19] Deci EL, Ryan RM. Self-determination theory: A macrotheory of human motivation, development, and health. Canadian Psychology. 2008; 49: 182-185.

[20] Standage M, Ryan RM. Self-determination theory and exercise motivation: Facilitating self-regulatory processes to support and 


\section{World Journal of Research and Review (WJRR) \\ ISSN:2455-3956, Volume-8, Issue-6, June 2019 Pages 06-15}

maintain health and well-being. In Roberts GC, Treasure DC, editors. Advances in Motivation in Sport and Exercise (pp. 233-270). Champaign, IL: Human Kinetics. 2012.

[21] Weiss MR, Amorose AJ. Motivational orientations and sport behavior. In Horn T.S. Editor. Advances in Sport Psychology (pp. 115-155). Champaign, IL: Human Kinetics. 2008.

[22] Amorose AJ, Anderson-Butcher D. Autonomy-supportive coaching and self-determined motivation in high school and college athletes: A test of self-determination theory. Psychology of Sport and Exercise. 2007; 8: 654-670.

[23] Goodwin D, Fitzpatrick D, Thurmeier R, Hall C. The decision to join Special Olympics: Parent's perspectives. Adapted Physical Activity Quarterly. 2006; 23: 163-183.

[24] Rossow-Kimball B, Goodwin D. Self-determination and leisure experiences of women living in two group homes. Adapted Physical Activity Quarterly. 2009; 26: 1-20.

[25] Rouse P, Duda J, Ntoumanis N, Jolly K, Williams G. (2016). The development and validation of the Interpersonal Support in Physical Activity Consultations Observational Tool. European Journal of Sport Science. 2016; 16: 106-114.

[26] Pannekoek L, Piek J, Hagger M. The children's Perceived Locus of Causality Scale for physical education. Journal of Teaching in Physical Education. 2014; 33: 162-185.

[27] Vlachopoulos S, Michailidou S. Development and initial validation of a measure of autonomy, competence, and relatedness in exercise: The basic Psychological Needs in Exercise Scale. Measurement in Physical Education and Exercise Science. 2006; 10: 179-201.

[28] Vlachopoulos S, Katartzi E, Kontou M. The Basic Psychological Needs in Physical Education Scale. Journal of Teaching in Physical Education. 2011; 30: 263-280.

[29] Wilson P, Rogers W, Rodgers W, Wild T. The Psychological Need Satisfaction in Exercise Scale. Journal of Sport and Exercise Psychology. 2006; 28: 231-251.

[30] Gillet N, Vallerand R, Paty E, Gobance L, Berjot S. French validation and adaptation of the perceived autonomy support scale for exercise settings to the sport context. International Journal of Sport and Exercise Psychology. 2010; 8: 117-128.

[31] Gillet N, Vallerand R, Rosnet E. Motivational clusters and performance in a real-life setting. Motivation and Emotion. 2009; 33: 49-62.

[32] Markland D, Tobin V. A modification of the behavioral regulation in exercise questionnaire to include an assessment of amotivation. Journal of Sport \& Exercise Psychology. 2004; 26: 191-196.

[33] Griffin K, Wang E, Hart M. Measuring fitness actions and dispositions associated with physical activity: Validation of a self-report instrument The Physical Educator. 2014; 71: 1-15.

[34] Ryan RM, Connell JP. Perceived locus of causality and internalization: Examining reasons for acting in two domains. Journal of Personality and Social Psychology. 1989; 57: 749-761.

[35] Tsalavoutas I, Reid G. Competence satisfaction: Risk taking and achievement. Adapted Physical Activity Quarterly. 2006; 23: 410-423.

[36] Orr K, Tamminen K, Sweet S, Tomasone J, Arbour-Nicitopoulos K. 'I've had bad experiences with team sport'. Sport-participation, peer need-thwarting, and need supporting behaviors among youth identifying with physical disability. Adapted Physical Activity Quarterly. 2018; 35: 36-56.

[37] Perreault S, Vallerand R. A test of Self-determination theory with wheelchair basketball players with and without disability. Adapted Physical Activity Quarterly. 2007; 24: 305-316.

[38] .Powers L, Turner A, Ellison R, Matuszewski J, Wilson R, Phillips A, Rein C. A multi-component intervention to promote adolescent self-determination. Journal of Rehabilitation. 2001; 67: 13-19.

[39] Banack HR, Sabiston CM, Bloom GA. Coach autonomy support, basic need satisfaction, and intrinsic motivation of Paralympic athletes. Research Quarterly for Exercise and Sport. 2011; 82: 722-730.

[40] Deci EL, Hodges R, Pierson L, Tomassone J. Autonomy and competence as motivational factors in students with learning disabilities and emotional handicaps. Journal of Learning Disabilities. 1992; 25: 457-471.

[41] Pan C. School time physical activity of students with and without autism spectrum disorders during PE and recess. Adapted Physical Activity Quarterly. 2008; 25: 308-321.

[42] Todd T, Reid G, Butler-Kisber L. Cycling for students with ASD: Self-regulation promotes sustained physical activity. Adapted Physical Activity Quarterly. 2010; 27: 226-241.

[43] Carlsson K. Handling dilemmas of self-determination in 'user-centred' rehabilitation. Disability \& Rehabilitation. 2007; 29: 245-253.

[44] Sexton E, O’Donovan M, Mulryan N, McCallion P, McCarron M. Whose quality of life? A comparison of measures of self-determination and emotional wellbeing in research with older adults with and without intellectual disabilities. Journal of Intellectual and Developmental Disability. 2016; 41: 324-337.

[45] Pozeriene J, Adomaitiene R, Ostaseviciene V, Reklaitiene D, Kragniene I. Sport participation motivation of athletes with intellectual disabilities. Uddymas Kuno Kultura Sports. 2008; 70: 69-75.

[46] Frielink N, Schuengel C, Embregts P. Autonomy support, need satisfaction, and motivation for support among adults with intellectual disability: Testing a self-determination theory model. American Journal of Intellectual and Developmental Disabilities. 2018; 123: 33-49.

[47] Reid G, Vallerand RJ, Poulin C, Crocker P. The development and validation of the pictorial motivation scale in physical activity. Motivation and Emotion. 2009; 33: 161-172.

[48] Sajute J. Orientation of participation motivation is physical activity for persons with intellectual disabilities. Unpublished master thesis. Kaunas: Lithuanian Academy of Physical Education. 2002.

[49] Deci EL, Ryan RM. The "what" and "why" of goal pursuits: Human needs and the self-determination of behavior. Psychological Inquiry. 2000; 11: 227-268.

[50] Yun J, Ulrich D. Estimating measurement validity: A tutorial. Adapted Physical Activity Quarterly. 2002; 19: 32-47.

[51] Thomas J, Nelson J. Research methods in physical activity 2nd ed. Champaign, IL: Human Kinetics. 1996.

[52] Thompson B, Vacha - Haase T. Psychometrics is datametrics: the test is not reliable. Education and Psychology Measures. 2000; 60: 174-195.

[53] Weir J. Quantifying test-retest reliability using the Intraclass correlation coefficient and the SEM. Journal of Strength and Conditioning Research. 2005; 19: 231-240.

[54] Boyle G. Does item homogeneity indicate internal consistency or item redundancy in psychometric scales? Personality and Individual Differences. 1991; 12: 291-294.

[55] Reid G, Vallerand RJ, Poulin C. Pictorial motivation scale. Unpublished Scale, McGill University, Canada. 2001.

[56] American Psychiatric Association. Diagnostic and statistical manual of mental disorders. 5th ed. Arlington, VA: American Psychiatric Publishing. 2013.

[57] Thompson JR, Bradley V, Buntinx W, Schalock RL, Shogren KA, Snell ME, Yeager MH. Conceptualizing supports and the support needs of people with intellectual disability. Intellectual and Developmental Disabilities. 2009; 47: 135-146.

[58] ICF (2001). WHO ICF: International Classification of Functioning Disability and Health. Available at: http://www.who.int/classifications/icf/en/. Assessed on: 18 November 2011.

[59] Reynolds - Keefer L, Johnson R. Creating effective questionnaires with pictures. Practical Assessment, Research \& Evaluation. 2011; 16(8): 1-7.

[60] Vallerand, RJ. Toward a hierarchical model of intrinsic and extrinsic motivation. In. Zanna MP Editors. Advances in experimental social psychology (Vol. 29, pp. 271-360). New York, NY: Academic Press. 1997.

[61] Vallerand RJ, Deci EL, Ryan RM. Intrinsic motivation in sport. In Pandoff KB, Editors. Exercise and sport science reviews (pp. 389-425). New York: Macmillian. 1987.

[62] Vallerand RJ, Reid G. Motivation and special populations: Theory, research, and implications regarding motor behavior. In Reid G Editors. Problems in movement control (pp. 159-197). Amsterdam: North-Holland. 1990.

[63] Wehmeyer M. Student self-report measure of self-determination for students with cognitive disabilities. Education and Training in Mental Retardation and Developmental Disabilities. 1996; 45: 282-293.

[64] US Census Bureau, Census Bureau Guideline. Language Translation of Data Collection Instruments and Supporting Materials. Census Bureau $\begin{array}{llll}\text { Website. Accessed } & \text { March } & \end{array}$ http://www.census.gov/cac/www/007585.html

[65] Li H, Lopez V. Children's emotional manifestation scale: Development and testing. Journal of Clinical Nursing. 2005; 14: 223-229.

[66] Shrout P. Measurement reliability and agreement in psychiatry. Statistical Methods in Medical Research. 1998; 7: 301-317.

[67] Cohen J. Statistical power analysis for the behavioral sciences.2nd ed. Upper Saddle River NJ: Prentice - Hall Inc. 1998.

[68] Grimm, L. Statistical applications for the behavioral sciences. New York, NY: John Wiley and Sons, Inc. 1993.

[69] Croce RV, Horvat M, McCarthy E. Reliability and concurrent validity of the Movement Assessment Battery for Children. Perceptual and Motor Skills. 2001; 93: 275-280. 
[70] Mageau GA, Vallerand RJ. The coach-athlete relationship: a motivational model. Journal of Sport Sciences. 2003; 21: 883-904.

[71] Hutzler Y, Korsensky O. Motivational correlates of physical activity in persons with an intellectual disability: A systematic literature review. Journal of Intellectual Disability Research. 2010; 54: 767-786.

[72] Chatzisarantis N, Hagger M. Mindfulness and the intention-behavior relationship within the theory of planned behavior. Personality and Social Psychology. 2007; 33: 663-676.

[73] Trost S, Owen N, Bauman A, Sallis J, Brown W. Correlates of adults' participation in physical activity: review and update. Medicine and Science in Sports and Exercise. 2002; 34: 1996-2001.

[74] Wehmeyer M, Bolding N. Enhanced self-determination of adults with intellectual disabilityas an outcome of moving to community-based work or living environents. Journal of Intellectual Disability Research. 2001; 45: 371-383.

[75] Buttimer J, Tierney E. Patterns of leisure participation among adolescents with a mild intellectual disability. Journal of Intellectual Disability. 2005; 9: 25-42.

[76] Temple V, Frey G, Stanish H. Physical activity of adults with mental retardation: Review and research needs. American Journal of Health Promotion. 2006; 21: 2-12.

[77] O'Brien G. Dual diagnosis in offenders with intellectual disability: setting research priorities: a review of research findings concerning psychiatric disorder (excluding personality disorder) among offenders with intellectual disability. Journal of Intellectual Disability Research. 2002; 46: 21-30.

[78] Robertson J, Emerson E, Gregory N, Hatton C, Turner S, Kessissoglou S, Hallam A. Lifestyle related risk factors for poor health in residential settings for people with intellectual disability. Research in Developmental Disabilities. 2000; 21: 469-486.

[79] Nunually J. Psychometric theory. New York, NY: McGraw-Hill. 1967.

[80] .Poulin C. A pictorial motivation scale in physical activity for people with a mild intellectual disability. Unpublished master thesis, McGill University, Montreal. 1992.

\section{Author Profile}

Dr Skordilis Emmanouil is the contact person and is employed as an Associate Professor in the School of Physical Education and Sport Science, in the National and Kapodistrian University of Athens. Dr Skordilis specializes in the field of Adapted Physical Activity, and teaches relevant courses such as 'Inclusive Physical Education', 'Measurement and Evaluation in Adapted Physical Activity', Adapted Physical Activities for All' etc. He has published several research articles in the field, and participated in international congresses addressing, among others, the effect of physical activity for individuals with developmental and physical disabilities.

Dr Greenlees Iain is a Professor of Sport Psychology at the Chichester Institute of Sport, University of Chichester, UK. Professor Greenlees specializes in the field psychology of sport and coaching and has published several research articles on coaching psychology, motivation and well-being. He has also published research developing measurement tools in sport psychology.

Dr Chrysagis Nikolaos is a research assistant in the School of Physical Education and Sport Science, in the National and Kapodistrian University of Athens. Dr Chrysagis specializes in the field of Adapted Physical Activity and Physiotherapy. He has published several research articles addressing, the effect of physical activity and physiotherapy for individuals with developmental and physical disabilities

Dr Eirini Grammatopoulou is a Professor at the Physiotherapy Department, University of West Attica in Athens, and head of the Physiotherapy Department. She is the author of 25 publications in international peer-reviewed journals in the field, has authored seven books and 10 book chapters in the past and teaches courses relevant to the 'Adapted Physical Activity' and 'Physiotherapy' fields.

Papadopoulou Vasiliki, M.Sc. is a physical educator specializing in the 'Adapted Physical Activity' field. She is actively involved in several programs addressing the involvement of individuals with developmental disabilities in exercise and daily physical activities.

Dr Pardo Gil Francisco Javier is an associate professor in the Biomedical Sciences Department, in the Universidad Europea de Madrid. Dr. Pardo specializes in the field of Sport Sciences and teaches Human Physiology and
Exercise Physiology courses. He has published several articles in the field of physiological adaptations to exercise.

Dr Gaillard Joel is a Professor in the University of Lorraine, France. His teaching and research work focuses in the development of skills, vocational training and career of future teachers and coaches of individuals with disabilities.

Skordilis Antonios is a member of Amfidromo Chorotheatro and co-director of Egomio Cultural Centre in Nicosia. He is a mechanical engineer, journalist specialized for many years (since 1996) in the field of People with Disabilities and author of the National Greek Guide "Disability and Journalism". Since 2000 he is a consultant of the most important disability magazines in Greece and Cyprus, and has published several articles in the field (Presspublica/Greece, Egomio blog/Cyprus).

Dr Dias Joao is a visiting Professor and researcher for the Associacao para a Recuperacao de Cidadaos Inadaptados da Lousa - ARCIL, in Portugal. He specializes in the field of disability and inclusion, rehabilitation services and professional training for individuals with disabilities and their families.

Serras Dionysios, M.Sc, is a psychologist, specializing in sports \& exercise psychology. He is actively involved in the development and implementation of several physical activity programs for young adults with intellectual disabilities, autism and psychiatric disorders. He is presently the manager of the Erasmus+ "Alive \& Kicking" Intervention \& research Project. 